\title{
Acquired Factor V Inhibitor with Symptoms and Titer of Inhibitor Differences: Report of Two Cases
}

\author{
Fanfan Li Kuangyi Shu Jie Liu Chenfang Shen Xiaoou Wang Zhaohua Zhang \\ Minghua Jiang
}

Clinical Laboratory Center, The second Affiliated Hospital and Yuying Children's Hospital of Wenzhou Medical University, Wenzhou, China

Dear Editor,

A 51-year-old man with normal coagulation underwent surgery because of skull trauma. After the procedure, ceftriaxone was intravenously administered at $2.0 \mathrm{~g} \mathrm{ev}$ ery $12 \mathrm{~h}$ to prevent a postoperative infection. Nevertheless, computed tomography revealed a pulmonary infection 2 weeks later. Consequently, he was treated with an extra antibiotic ceftazidime. In the 21 days of anti-infective treatment, the patient was found to have oral mucosal bleeding and abnormal coagulations: the activated partial thromboplastin time (APTT) was 167.5 s (normal range 30-45 s) and the prothrombin time (PT) was $30 \mathrm{~s}$ (normal range $12-15 \mathrm{~s})$. His biochemical and immune examinations were normal (Table 1). Subsequently, he was injected with vitamin $\mathrm{K}$ (VK) and prothrombinase complex. However, there was no significant improvement in his coagulations even after transfusing fresh frozen plasma (FFP; Fig. 1a). Coagulation factor assay showed that the factor $\mathrm{V}$ activity was only 2\% (normal $50-150 \%$ ), FVII and FXII activity reduced slightly, while other coagulation factor activities were within normal limits (Table 1). A mixing study manifested that neither PT nor APTT could be corrected (Fig. 1c). The factor $\mathrm{V}$ inhibitor test was positive and showed the titer of inhibitor was 128 Bethesda units (BU). Thus, the patient was diagnosed with acquired factor $\mathrm{V}$ deficiency and the coagulation times reduced after injection with prednisone at $10 \mathrm{mg}$ for 2 days (Fig. 1a).

Another patient, a 78-year-old man with intracranial hemorrhage, was administered several months ago. His PT (normal range 12-15 s) and APTT (normal range $30-45 \mathrm{~s}$ ) were prolonged, at 23 and $61 \mathrm{~s}$, respectively. His biochemical and immunologic tests were both regular (Table 1), but brain computed tomography scanning showed a right temporal brain hemorrhage. After removal of intracranial hematoma with anything to correct coagulation, surgical wound bleeding was detected. Coagulation assay showed the factor $\mathrm{V}$ activity was only $13 \%$. Factor XII also reduced slightly (Table 1). After mixing with normal plasma (Fig. 2d), the prolonged PT and APTT were partially corrected. Furthermore, VK and FFP could not alleviate the coagulations (Fig. 1b). Thus, we suspected that the abnormity was due to the factor $\mathrm{V}$ inhibitor, which was later confirmed by the Bethesda method. Although the patient only had a low titer of inhibitor (2 BU), his bleeding symptoms were obvious. However, we did not provide other treatment than FFP, and the patient was discharged 2 months later without symptoms. As with the first case described above, we did not find any mutation at the factor $\mathrm{V}$ gene in this case.

Acquired factor $\mathrm{V}$ deficiency is mainly caused by a factor $\mathrm{V}$ inhibitor, which results in low factor $\mathrm{V}$ activity and is associated with various factors, such as antibiotics [1], surgery, malignancy [2], autoimmune disease, blood transfusions, and exposure to bovine thrombin [3]. In the study by Franchini and Lippi [4], antibiotics like $\beta$-lactam aminoglycosides showed the most common risk, accounting for $42 \%$ of the risk cases, yet surgery and infection accounted for 31 and 23\%, respectively. This may offer an interpretation to the first
KARGER

(c) 2019 S. Karger AG, Basel

E-Mail karger@karger.com

www.karger.com/aha
Minghua Jiang

Clinical Laboratory Center, The second Affiliated Hospital and Yuying Children's Hospital of Wenzhou Medical University 109 Xueyuan West Road, Wenzhou, Zhejiang 325027 (China) E-Mail minghua93@126.com 
Table 1. Laboratory findings

Case 1

Complete blood count

$\mathrm{WBC}, \times 10^{9} / \mathrm{L}$

$\mathrm{RBC}, \times 10^{12} / \mathrm{L}$

$\mathrm{Hb}, \mathrm{g} / \mathrm{L}$

HCT

$\mathrm{MCV}, \mathrm{fL}$

$\mathrm{MCH}, \mathrm{pg}$

PLT, $\times 10^{12} / \mathrm{L}$

Coagulation test

$\mathrm{PT}, \mathrm{s}$

PT-INR

APTT, s

Fbg, g/L

D-dimer

Coagulation factor assay

Factor II, \%

Factor V, \%

Factor VII, \%

Factor VIII, \%

Factor IX, \%

Factor X, \%

Factor XI, \%

Factor XII, \%

Factor V inhibitor, BU

Biochemistry

AST, U/L

ALT, U/L

$\gamma$-GTP, U/L

T-Bil, $\mu \mathrm{mol} / \mathrm{L}$

BUN, $\mathrm{mmol} / \mathrm{L}$

$\mathrm{Cr}, \mu \mathrm{mol} / \mathrm{L}$

\section{Immuno findings}

Antinuclear antibody

Anticardiolipin IgG

Lupus AC

$$
\begin{aligned}
& 9.1(4-10) \\
& 3.31(4-5.5) \\
& 98 \downarrow(120-150) \\
& 0.31 \downarrow(0.4-0.54) \\
& 93.9(80-100) \\
& 29.6(26-32) \\
& 373 \uparrow(100-300)
\end{aligned}
$$

Case 2

$$
\begin{aligned}
45.6 & \uparrow(12-15) \\
4.82 & \uparrow(0.85-1.15) \\
167.5 & \uparrow(30-45) \\
5.97 & \uparrow(2-4) \\
0.83 & \uparrow(0-0.5)
\end{aligned}
$$

$$
\begin{gathered}
6.0(4-10) \\
3.42(4-5.5) \\
108(120-150) \\
0.325(0.4-0.54) \\
95(80-100) \\
31.6(26-32) \\
141(100-300)
\end{gathered}
$$

$\uparrow$ and $\downarrow$ indicate values higher and lower than normal ranges, respectively. Normal ranges are shown in parentheses.

case reported here. Many new factors have also been reported as being related to factor $\mathrm{V}$ inhibitors, such as warfarin [5], amiodarone [6], dabigatran etexilate [7], and liver transplantation. However, we did not know why hemorrhage and factor $\mathrm{V}$ inhibitor emerged without any determined risks in case 2 . The mechanism behind the production of inhibitors was a mystery.

The clinical symptoms of patients with factor $\mathrm{V}$ inhibitor can range from asymptomatic hematological laboratory abnor-

Factor V Inhibitor with Symptoms and Titer of Inhibitor Differences malities to life-threatening hemorrhage. Remarkably, Franchini and Lippi [4] also referred to 4 cases associated with thrombotic complications instead of hemorrhage. In our cases, the first had $2 \%$ factor $\mathrm{V}$ activity and a high inhibitor titer, but only suffered oral mucosal bleeding, while the other case, with $13 \%$ factor V activity, presented serious hemorrhage. We speculated that this was owing to the detecting principles. The laboratory tests are based on poor platelet plasma, ignoring the factor
$\mathrm{V}$ derived from platelets. Nevertheless, the factor $\mathrm{V}$ derived from platelets is also pivotal and has sufficient capability to ensure the normal coagulation function. Thus, factor activity cannot exactly reflect a patient's actual clotting status [8]. Besides, antibiotics, surgery, and infection may have resulted in the high titer of inhibitor that emerged in case 1 , but we could not find these risks in case 2. In other words, the inhibitor resulting from drugs and stimulus probably caused mild symptoms, 


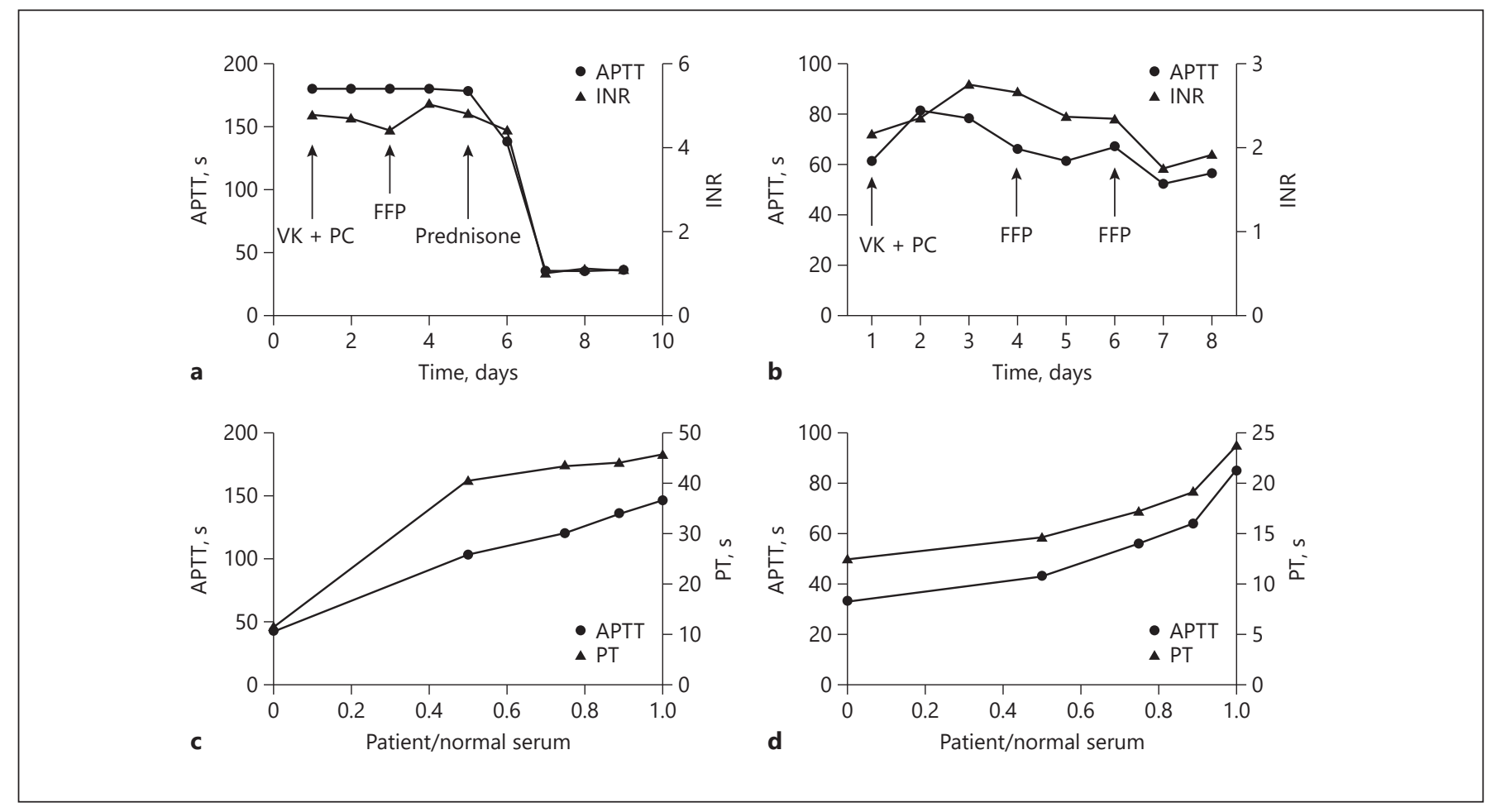

Fig. 1. INR (International Sensitivity Index) and APTT profiles of case 1 (a) and case 2 (b). Mixing test: sera from patient 1 (c) and patient 2 (d) and a healthy normal subject were mixed at the indicated ratios.

while the inhibitor generating spontaneously or caused by unknown reasons could cause a serious manifestation.

Herein, we have reported 2 rare cases of acquired factor $\mathrm{V}$ inhibitor that were comparable but differently induced, probably leading to opposite hemorrhagic manifestations.

\section{References}

1 Siekańska-Cholewa A, Jarosz A, Góralczyk T, Iwaniec T, Węgrzyn W, Drwiła R, et al. Acquired factor $\mathrm{V}$ inhibitor in a woman following aortic aneurysm surgery. Blood Coagul Fibrinolysis. 2014 Jul;25(5):515-7.

2 Aljohani NI, Matthews JH. Acquired factor V inhibitor in a patient with mantle cell lymphoma presenting with hematuria followed by thrombosis: a case report. Int Med Case Rep J. 2014 Feb;7:27-30.

3 Streiff MB, Ness PM. Acquired FV inhibitors: a needless iatrogenic complication of bovine thrombin exposure. Transfusion. 2002 Jan; $42(1): 18-26$.

\section{Statement of Ethics}

The patients gave their informed consent for the writing of this letter.

4 Franchini M, Lippi G. Acquired factor V inhibitors: a systematic review. J Thromb Thrombolysis. 2011 May;31(4):449-57.

5 Gartrell B. Acquired factor V inhibitor complicating warfarin therapy. Am J Hematol. 2011 Aug;86(8):710-2.

6 Shreenivas AV, Lipshitz J, Patel D. A rare case of factor $\mathrm{V}$ inhibitor in a patient on amiodarone therapy. Blood Coagul Fibrinolysis. 2012 Jun;23(4):342-4.

\section{Disclosure Statement}

The authors have no conflicts of interest to declare. The work has no funding sources.

7 Sekiguchi Y, Yoshikawa H, Shimada A, Imai $\mathrm{H}$, Wakabayashi M, Sugimoto K, et al. Acquired factor $\mathrm{v}$ inhibitor developing after treatment with dabigatran etexilate methanesulfonate: a case report and review of the literature. Indian $\mathrm{J}$ Hematol Blood Transfus. 2014 Sep;30(S1 Suppl 1):275-9.

8 Lipshitz J, Chelliah T, Aledort L. A case of factor $\mathrm{V}$ inhibitor with complete correction of the PT and aPTT upon mixing. Am J Hematol. 2012 Mar;87(3):313-5. 